ARTICLE

\title{
Design of Active-Type Personal Dosemeter for High-Energy Neutrons
}

\author{
Hirotaka RITO*, Kensuke IWANO, Tomoya YAMAUCHI, Keiji ODA \\ Graduate School of Maritime Sciences, Kobe University,5-1-1 Fukae-minamimachi, Higashinada-ku, Kobe 658-0022, Japan
}

\begin{abstract}
In order to design an active-type personal dosemeter having sufficient sensitivity to high-energy neutrons up to $100 \mathrm{MeV}$, the characteristic response of several types of silicon ( $\mathrm{Si}$ ) semiconductor detectors has been investigated experimentally and theoretically. At first, the response of each detector has been checked to protons accelerated by a Van de Graff accelerator installed at Kobe University. A fully-depleted Si detector was selected as a promising detector element owing to a simple structure and comprehensive characteristics. In the next step, neutron irradiation experiments were carried out in a monoenergetic reference field in JAEA. On the other hand, theoretical distributions of the deposited energy were estimated in a simplified geometry consisting of Si and PE layers. An agreement in the relative shape was confirmed between the theoretical and experimental results. Applying this calculation procedure to other neutron energies and various thickness of sensitive layer, it was found that the energy dependence could be improved by the two-window technique by setting different discrimination levels on the pulse height. Another important characteristic of the angular dependence was also discussed for practical application of a personal dosemeter for high energy neutrons.
\end{abstract}

KEYWORDS: active-type personal dosemeter, energy dependence, two-window technique, angular dependence

\section{Introduction}

Recently, a large number of accelerator facilities has been applied to fruitful fields of fundamental, medical, material, environment sciences, and so on. In Japan, a MW-class proton accelerator facility J-PARC (Japan-Proton Accelerator Research Complex) has been developed with collaborative big project of JAEA (Japan Atomic Energy Agency) and KEK (High Energy Accelerator Research Organization). With increasing the utilization of various neutron fields, monitoring of high-energy neutrons becomes more important from a viewpoint of radiation protection because most detectors are less sensitive to energetic neutrons, which leads to underestimation of the dose equivalent of radiation workers.

The personal dosemeters for radiation protection dosimetry are, in general, classified into two types according to their responsivity. The passive type dosemeters are used for measuring the total dose integrated over a period, typically one month. A plastic nuclear track detector such as poly-allyl diglycol carbonate (PADC, commercially "CR-39") is one of most popular elements, and for which several techniques have been proposed and checked for improving the sensitivity to high-energy neutrons. ${ }^{1-2)}$ For example, a two-layer structure consisting of polyethylene and deuterized hydrocarbon sheets has been proposed as a promising radiator to enhance the sensitivity to high-energy neutrons up to several tens of $\mathrm{MeV}$. Another attempt has also been tried based on careful observation of microscopic pictures to obtain additional information such as distribution in the etch-pit size, gray level and shape factor.

On the contrast to these activities to passive-type dosemeters, few improvement has been tried in recent years

*Corresponding Author, Tel. \& Fax. +81-78-431-6304,

E-mail:092w525w@stu.kobe-u.ac.jp on active-type dosemeter that can display the dose rate or accumulated dose in real time. After surveying the performance of several prevailing detectors including a super heated liquid-drop detector, an electronic personal dosemeter composed of silicon ( $\mathrm{Si}$ ) semiconductor detector and a thin polyethylene sheet is most promising for practical application to high-energy neutron dosimetry ${ }^{3)}$. In the previous report ${ }^{4}$, the characteristic responses of a commercial EPD (Electronic Personal Dosemeter) and other Si detectors have been checked in mono-energetic reference fields, where neutrons were normally incident on the detectors. It is the purpose of this study to make an attempt for improving energy dependence of the dosemeter and to estimate its dependence on the angle of neutron incidence.

\section{Calculation Method}

It is well-known that a Monte Carlo simulation is most effective technique to predict the detector response exposed to indirectly ionizing radiation. It requires, however, much time to obtain the result with a satisfactorily high statistical precision for a large number of combinations of several parameters. So, we introduce a mathematical model for numerical calculations, which is convenient for designing or optimizing the parameters.

For simplicity, a contribution of neutron interaction with carbon and influence by gamma-rays accompanying a neutron field are neglected in a geometry of two layers of Si and polyethylene (PE) as shown in Figure 1, where neutrons with an energy, $E_{n}$ are incident on PE layer with an angle, $\psi$ with respect to the line normal. The number of protons recoiled per unit solid angle at a depth $z$ is $N d \sigma / d \Omega$, where $N$ is the density of hydrogen atoms in $\mathrm{PE}$ and $d \sigma / d \Omega$ is the angular differential cross section. Let the recoil angle be $\theta$, then the initial kinetic energy of protons, $E_{p}$ is $E_{n} \cos ^{2} \theta$, and the angle of proton trajectory, $\alpha$ is expressed by the following relation: 
$\cos \alpha=\cos \theta \cos \psi-\sin \theta \sin \psi \cos \varphi$

where $\varphi$ is the azimuthal angle. Using the range-energy relation $R(E)$ for protons, the energy $E_{i n}$ with which the proton enters into Si layer, is theoretically found to be the value of proton energy corresponding to the range of $R_{P E}\left(E_{i n}\right)=R_{P E}\left(E_{p}\right)-z / \cos \alpha$. In a similar way, the energy with which the proton leave the Si layer, $E_{\text {out }}$ is estimated to be such a value that its range $R_{S i}\left(E_{\text {out }}\right)$ is equivalent to $R_{S i}\left(E_{i n}\right)-W_{d} / \cos \alpha$, where $W_{d}$ is the thickness of Si layer. Then, the deposited energy, $E_{d}$ is obtained by the difference between $E_{\text {in }}$ and $E_{\text {out }}$. Integrating over all directions and all positions in $\mathrm{PE}$, a distribution of the deposited energy, $P\left(E_{d}\right)$ is expressed by

$$
P\left(E_{d}\right)=\frac{d}{d E_{d}}\left[N \int_{0}^{W_{r}} \int_{0}^{\frac{\pi}{2}} \int_{0}^{2 \pi} \frac{d \sigma}{d \Omega} d \varphi d \theta d z\right]
$$

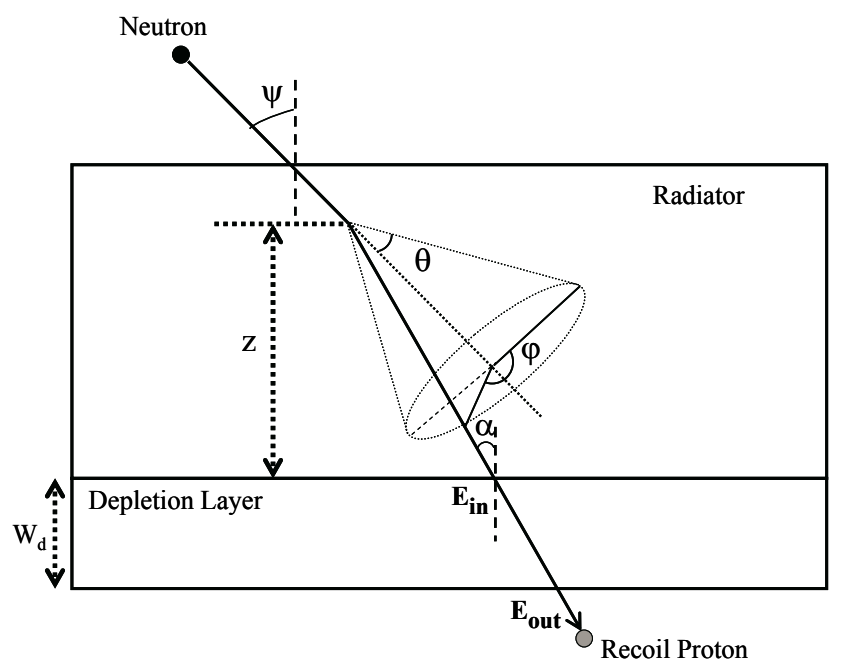

Fig.1 Geometrical relation in analytical calculation.

\section{Characteristic Response of Si Detector with Polyethylene Radiator}

A Si detector practically applicable to a personal dosemeter for radiation workers have several requirements of compact size, light weight, stable operation, less influence by gamma-rays, and so on. After literature research, we nominated and preliminarily checked three types of $\mathrm{Si}(\mathrm{Li})$ of $1 \mathrm{~mm}$ thick, p-n junction type and fully-depleted one. The first one was found to be affected considerably by gamma-rays. As to the second type detector, questionable response to charged particle was observed, which may be caused by charges generated outside of the depletion layer, namely Funneling effect. ${ }^{5}$ So, the third one, a fully-depleted type surface barrier detector (RAYTEC Co. Ltd., Japan, $30-025-20 \mathrm{~B}$ ) having the sensitive area of $0.25 \mathrm{~cm}^{2}$ was selected as one of promising elements. Its characteristic response to protons was investigated using a Van de Graaff accelerator installed at Kobe University. The depletion layer of $20 \mu \mathrm{m}$ thick is obtained by an applied voltage of $10 \mathrm{~V}$ according to the operation manual. In order to check this nominal thickness, the pulse height was measured for different angles of incidence of $2.95 \mathrm{MeV}$ protons, which were scattered by a thin gold foil in a vacuum chamber. The signal generated was lead to MCA through a preamplifier (ORTEC 142H) and a linear amplifier (ORTEC 572). The channel number was converted into the energy through a calibration experiment using an ${ }^{241} \mathrm{Am}$ alpha-source and a pulse generator. The results are shown in Fig. 2, where measured values represented by dots exceed predicted one shown by a broken line. The difference may be attributed mainly to incorrect energy calibration or thicker sensitive layer of Si detector.

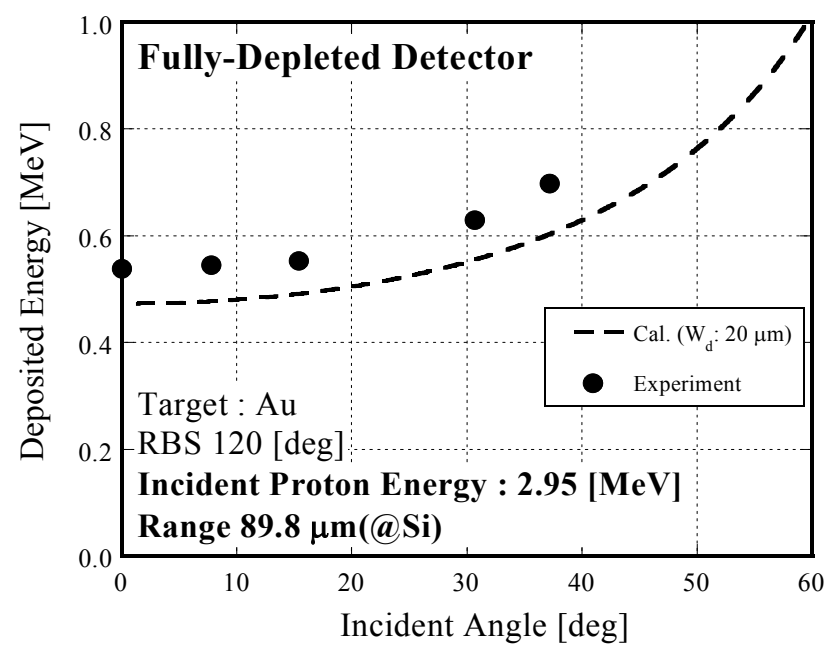

Fig.2 The deposited energy for several angles of incidence of $2.95 \mathrm{MeV}$ protons.

In the next step, neutron irradiation experiment has been carried out at Fundamental Radiation Sources (FRS), Japan Atomic Energy Agency. Mono-energetic neutrons of 5.0 $\mathrm{MeV}$, generated by $\mathrm{D}-\mathrm{D}$ reactions were incident normally on the detector with a PE radiator of $350 \mu \mathrm{m}$ thick. The measured pulse height distribution is shown in Fig. 3 by a solid line, where abscissa was calibrated in the same manner as stated above and the ordinate is the absolute value of the counts per unit sensitive area per unit neutron fluence. A dotted line in this figure represents the distribution in the deposited energy calculated numerically with eq. (2). The shapes of both distributions are relatively similar to each other, but the absolute value and the peak position dose not agree well. In order to confirm the validity of numerical calculations, a Monte Carlo code, PHITS ${ }^{6-7)}$, was employed, and the result is shown in Fig. 3 by a broken line. Very good agreement was found between two calculation results. It is concluded that a shift to higher energy side of the measured distribution might be ascribed to the problem in the energy calibration and/or deviation from the nominal thickness, as were stated above. The Landau distribution may be one of the responses for the difference between measured and calculated results.

The number of charged particles due to interaction with $\mathrm{Si}$ layer has also been calculated by using PHITS code. For example, the contribution of alpha particles was estimated to be about $0.4,1.0$ and $1.4 \%$ for neutron energies of 10,50 and $100 \mathrm{MeV}$, respectively. For simplicity, the numerical calculation method based on eq. (2) was intensively used for various parameters, rather than a time-consuming calculation with PHITS code, in latter sections. 


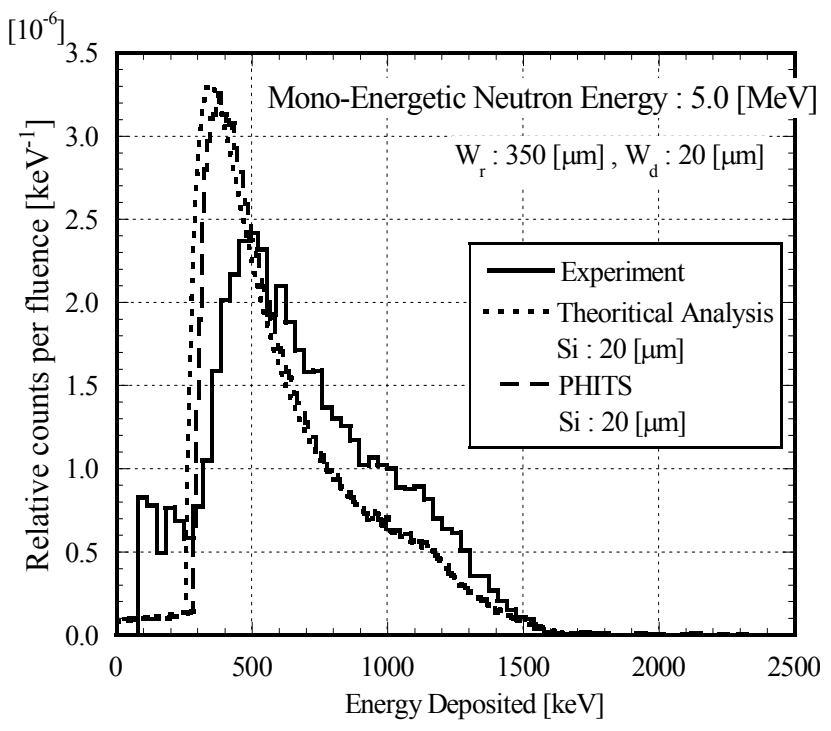

Fig.3 Comparison among the pulse height distributions obtained by theoretical calculation method, experiment and PHITS for $5.0 \mathrm{MeV}$ neutrons detected by a fully-depleted detector covered with a thin polyethylene radiator.

\section{Design of Personal Dosemeter}

\section{Energy Dependence}

An ideal neutron dosemeter should have not only high sensitivity but also good characteristics of the energy and incident angle dependences. In this section, an attempt of improvement in the energy dependence is discussed in a relatively higher region between 10 and $100 \mathrm{MeV}$, to which our concern is focused in this study.

The detection efficiency is, in our case of Si detector, expressed as the number of counted pulses per unit neutron fluence, which is the integral of the pulse height distribution between a lower and upper discrimination levels, $E_{L}$ and $E_{U}$. The detector sensitivity, $S$, is defined here as the efficiency divided by the conversion coefficient, $h_{p}$, from the neutron fluence into the personal dose equivalent, $H_{p}(10)$. Then,

$$
S\left(E_{n}\right)=\frac{1}{h_{p}\left(E_{n}\right)} \int_{E_{L}}^{E_{U}} P\left(E_{n}\right) d E_{d}
$$

the pulse height distribution, $P\left(E_{n}\right)$, is also a function of the detector thickness, $W_{d}$ or radiator one, $W_{r}$.

Typical results of calculation of eq. (3) are summarized in Fig. 4 under the condition that $E_{L}=120 \mathrm{keV}$ and $E_{U}=\infty$. A variation of the energy dependence with the radiator thickness is shown by lower three curves. It is found that the sensitivity can be increased with the radiator thickness, for a few tens of $\mathrm{MeV}$, but the enhancement by the radiator becomes less remarkable for high energies. An ideal dosemeter, has no dependence of the sensitivity on the neutron energy, namely the energy response curve should be a straight horizontal line. It is concluded after many calculations with possible combinations of two parameters that no drastic improvement could be achieved especially in the neutron energy up to several tens of MeV.

So, we have tried to apply the two-window technique, where the pulse height distribution is divided into two parts by setting respective discrimination levels and then a corrected response can be evaluated by a linear combination of both integrated values weighted by appropriate factors.

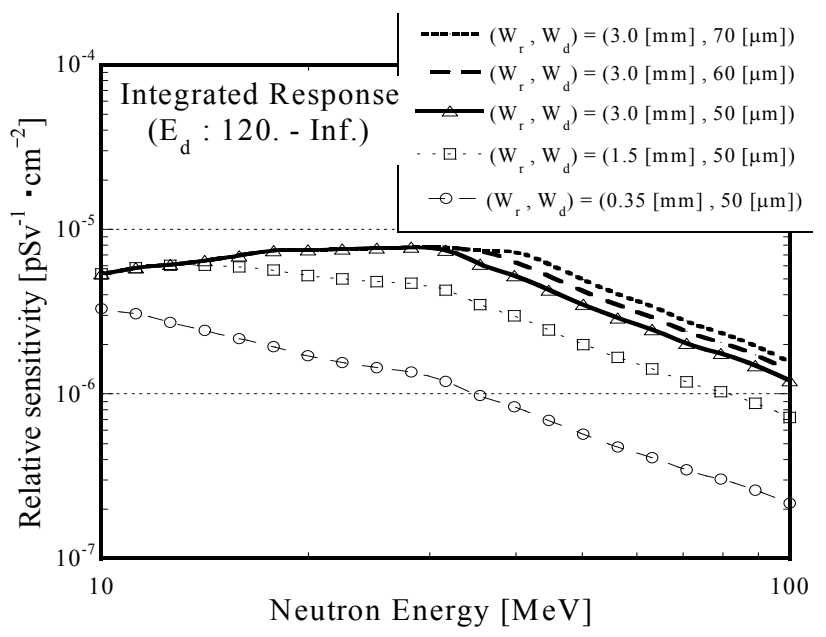

Fig.4 Dependence of the sensitivity on the neutron energy for various combinations of radiator and detector thicknesses.

Fig. 5 shows a process of improving the dosemeter sensitivity, where the detector and radiator thicknesses are assumed to be $60 \mu \mathrm{m}$ and $3 \mathrm{~mm}$, respectively. A gray solid line in Fig. 5 represents an original response curve with suppressing a gamma cut-off level of $120 \mathrm{keV}$. A dotted line and a broken one correspond to the energy dependence of the sensitivities, $S_{1}$ and $S_{2}$, windows of $120-300$ and $300-2500$ $\mathrm{keV}$, respectively. It is easily recognized from the figure that the partial sensitivity would have a different energy dependence according to a selection of the window. By taking a sum of $2.2 S_{1}+S_{2}$, the response curve can be improved, and the variation with the neutron energy between 10 and $100 \mathrm{MeV}$ could be reduced by $30 \%$, as is shown by a bold solid line in Fig. 5.

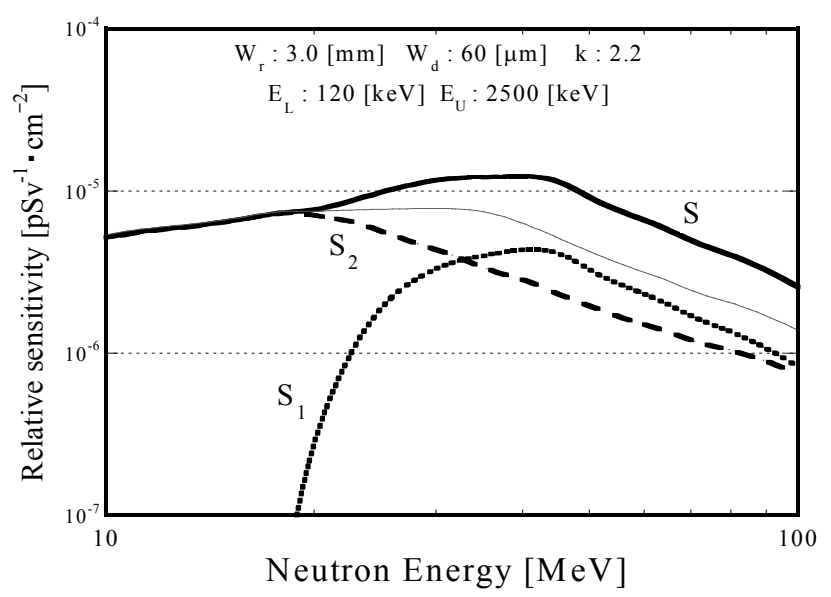

Fig.5 Optimized energy dependence in neutron energy between 10 and $100 \mathrm{MeV}$ using the two-window technique.

\section{Angular Dependence}

In actual radiation fields in working areas, neutrons come from various directions. In this section, we made a consideration of another important characteristic of the 
angular dependence. The same procedure was applied to numerical calculations of pulse height distributions for different angles of neutron incidence.

An example of the results is shown in Fig. 6, where the pulse height distributions are plotted for five different incident angles. With increasing the angle, the spectrum shifts to higher energy side because the average length of proton trajectory becomes longer.

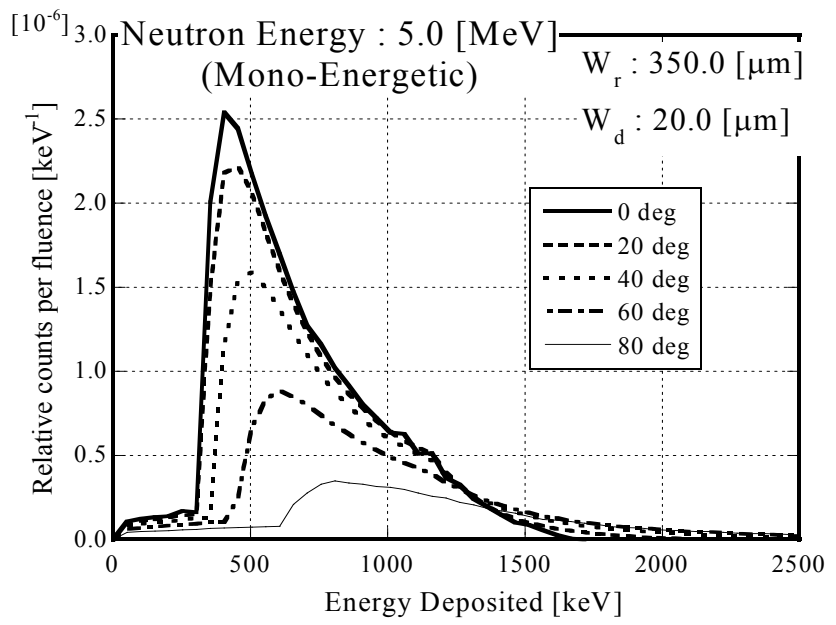

Fig.6 The pulse height distributions obtained in various angles for 5 $\mathrm{MeV}$ neutron incidence.

On the other hand, the number of pulses and the sensitivity decreases with the incident angle because of decrease of angular differential cross section. The angular dependence is shown in Fig. 7 for three neutron energies, where the sensitivity is normalized to that for normal incidence. About $40 \%$ or $60 \%$ reductions at 80 degree is considered to be inevitable so long as a plane-type detector is used. We are now trying to find a countermeasure based on the two-window technique or a specially designed radiator of multi-layer structure.

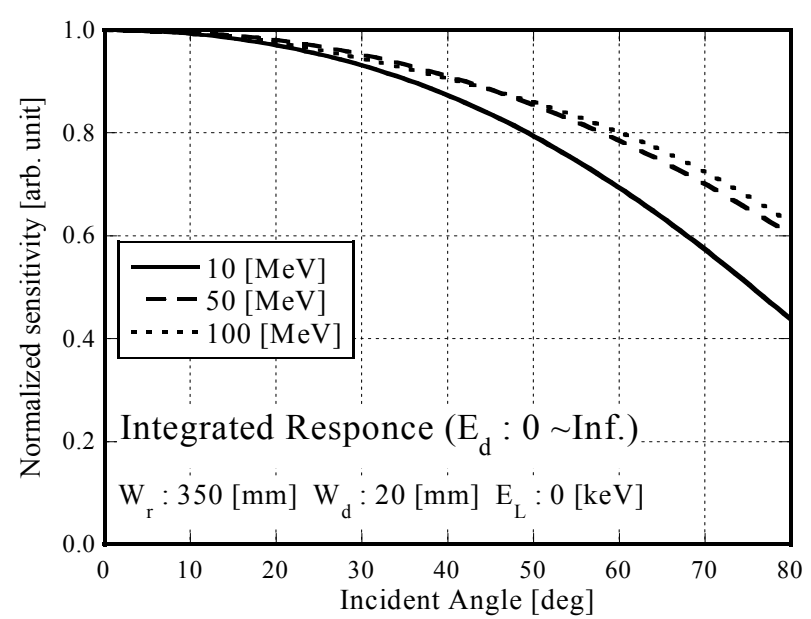

Fig.7 Angular dependence of the sensitivity normalized by the response for normal incident.

\section{Conclusion}

In this report, characteristic response of a fully-depleted $\mathrm{Si}$ detector to accelerated protons and mono-energetic neutrons was investigated for the purpose of improving an active-type personal dosemeter applicable to high energy neutrons.

The pulse height distribution of a $\mathrm{Si}$ detector with a polyethylene radiator was numerically obtained based on a simple mathematical model, which were compared with measured spectrum and PHITS calculation. The dosemeterensitivity, defined as the number of pulses per unit dose equivalent, has been calculated for various combinations of thickness of radiator and sensitive layer. Applying the two-window technique to the pulse height distributions, the energy dependence was successfully improved in the neutron energy between 10 and $100 \mathrm{MeV}$.

In the next step, we have discussed another important characteristic of incident angle dependence using the same calculation method, because the neutrons enter into the dosemeter from various directions in actual radiation fields such as a workplace around accelerators, on a high-flight altitude and in space ${ }^{8)}$. It was found that the sensitivities would be reduced to be $40 \%$ or $60 \%$ of that for normal incidence.

\section{Acknowledgement}

The authors would like to thank to all the staff of FRS facilities, JAEA for their kind help in neutron irradiation experiment.

\section{References}

1) K. Oda, Y. Imasaka, K. Tsukahara, et al., "Radiator effect on plastic nuclear track detectors for high-energy neutrons" Radiat. Meas., 36, 119-124 (2003).

2) K. Oda, Y. Imasaka, T. Yamauchi, et al., "Radiator design for detecting high energy neutrons with a nuclear track detector" radiat. Meas., 40, 570-574 (2005).

3) M. Luszik-Bhadra, "Electronic personal neutron dosemeter for high energies: measurments, new developments and further needs", Radiat. Prot. Dos., 126, 487-490 (2007).

4) K. Oda, K. Iwano, T. Yamauchi, et al., "Response of semiconductor-type electronic personal dosemeter to high-energy neutrons" Radiat. Meas., 43, 1072-1076 (2008).

5) T. Nakamura, T. Nunomiya, M. Sasaki, "Development of active environmental and personal neutron dosemeters", Radiat. Prot. Dos., 110, 169-172, 2004.

6) K. Niita, T. Sato, H. Iwase, et al., "PHITS-a particle and heavy ion transport code system" Radiat. Meas., 41, 1080-1090 (2006).

7) M. Luszik-Bhadra, M. Nakhostin, K. Niita, et al., "Electronic personal neutron dosemeters for energies up to $100 \mathrm{MeV}$ : Calculations using the PHITS code" Radiat. Meas., 43, 1044-1048 (2008)

8) D. T. Bartlett, L. G. Hager, R.J. Tanner, "The determination of the neutron component of cosmic radiation fields in spacecraft" Radiat. Meas., 36, 439-443 (2003). 